\title{
Caracterización por imágenes de los tumores óseos primitivos y metástasis óseas.
}

\section{Characterization by imaging of primitive bone tumors and bone metastases.}

\section{Elena Mariño Parra1* iD}

1. Servicio de Imagenología, Hospital Roberto Gilbert, Ministerio de Salud Pública del Ecuador, Guayaquil.
*Correspondencia:

emparra@hotmail.com

Teléfono [593] 042287310

Conflicto de intereses: La autora declara no tener conflictos de intereses.

Fondos: Ver la página 111

Recibido: 09 Octubre 2014 Aceptado: 02 Febrero 2016 Publicado: 30 Agosto 2017

Membrete bibliográfico: Parra E. Caracterización por imágenes de los tumores óseos primitivos y metástasis óseas. Rev. Oncol. Ecu 2017;27(2):105114.

DOI: https://doi.org/10.33821/178

Copyright Parrra. Este artículo es distribuido bajo los términos de Creative Commons Attribution License, el cual permite el uso y redistribución citando la fuente y al autor

\section{Resumen}

Introducción: La neoplasia ósea es una patología que altera la homeostasis del hueso dependiendo de la benignidad o malignidad del proceso. El estudio imagenológico aporta una serie de información que es fundamental para el diagnóstico y tratamiento de esta patología. El objetivo del presente reporte es establecer las características imagenológicas de los pacientes atendidos en el Servicio de Imágenes del Hospital "Juan Tanca Marengo" con tumores óseos.

Métodos: Se realizó un estudio observacional, en el que se registraron todos los casos con diagnóstico de neoplasias óseas atendidos en el Hospital "Juan Tanca Marengo", en Guayaquil entre enero del 2006 a enero del 2008. Para el análisis estadístico se emplearon medidas de tendencia central.

Resultados: Se incluyeron 100 casos, la edad promedio entre los pacientes con tumores óseos primarios fue menor que entre las metástasis (25.4 vs 32.8 años). Del grupo de pacientes con lesiones primarias fueron más frecuentes los del sexo masculino (56.0 vs $37.5 \%$ ). La afectación cortical fue mayor entre pacientes con tumor óseo metastásico que entre tumores primarios (62.5 vs 39.3\%). En las otras variables estudiadas no se presentaron diferencias significativas.

Conclusión: La mayoría de las características imagenológicas no son exclusivas del tipo de lesión ósea (primaria o metastásica). Las características de edad y género ayudan a la orientación diagnóstica de las imágenes de las lesiones tumorales óseas.

Palabras Claves: NEOPLASIA ÓSEA, METÁSTASIS, DIAGNÓSTICO, IMAGENOLOGÍA. 


\section{Abstract}

Introduction: The bone neoplasm is a pathology that alters the homeostasis of bone producing an alteration with different connotations depending on the benignity or malignancy of the process. The imaging study provides a series of information that is essential for the diagnosis and treatment of this pathology. The aim of this trial was to establish the characteristics of imaging of bone tumors in patients cared for in the Juan Tanca Marengo Hospital.

Methods: In Juan Tanca Marengo hospital from Guayaquil-Ecuador, conducted a study of descriptive observational retrospective in which were not randomly all cases of bone Neoplasms requiring imaging at the hospital in 2012. Statistical analysis: we used simple, relative frequencies, averages and deviation standard according to the characteristics of the variables.

Results: the mean age among patients with primary bone tumors was lower than among metastases (25.4 years vs. 32.8 years). Male patients were more frequent among patients with primary lesions (56, $0 \% 37.5 \%)$. Cortical involvement was higher among patients with bone tumor metastatic than between primary tumors (62.5\% vs $39.3 \%)$. Other characteristics did not differ.

Conclusion: The majority of the features imaging are not exclusive type (primary or metastatic) bone lesion. The characteristics of age and gender help diagnostic images of bone tumoral lesions orientation.

Keywords: BONE CANCER. BONE METASTASES. DIAGNOSIS. RADIOLOGY.

\section{Introducción}

La homeostasis del hueso se mantiene durante toda la vida principalmente por el equilibrio entre la actividad de los osteoclastos y osteoblastos que reabsorben el hueso y lo llenan de nueva matriz ósea. La ruptura de este equilibrio conduce a alteraciones cualitativas y cuantitativas del esqueleto. Entre las muchas causas de la interrupción de este equilibrio están las neoplasias óseas que incluyen los tumores benignos y malignos, anomalías focales reactivas, anormalidades metabólicas y, condiciones "neoplásicas" misceláneas que se desarrollan en el tejido óseo. En este ámbito la imagenología convencional tiene un rol principal como herramienta médica para la diferenciación de acuerdo a la apariencia radiológica [1-5]. También las técnicas de imagen de Tomografía Computarizada (TC) son útiles en la diferenciación [6], y en tercer lugar la Resonancia Magnética (RM), debido a que algunas lesiones benignas pueden ser sobreestimadas como resultado de la médula ósea y del edema de los tejidos blandos [7-9]. Las características radiológicas específicas que ayudan a evaluar el estudio imagenológico son la localización del tumor, los márgenes, la zona de transición, la reacción perióstica, la mineralización, tamaño y número de las lesiones y la presencia de un componente de los tejidos blandos.

Los avances en el diagnóstico y tratamiento de tumores, han aumentado la supervivencia de pacientes con cáncer en los últimos 30 años, lo que ha determinado el diagnóstico cada vez más frecuente de lesiones óseas. Como consecuencia de ello, el hueso ahora representa uno de los sitios más comunes de metástasis, después de pulmón e hígado. Por 
ejemplo, el $70 \%$ de los pacientes con cáncer de mama avanzado o carcinoma de próstata tienen metástasis óseas y están presentes en más del $90 \%$ de los pacientes que fallecen por estas patologías [10-12]. Esto significa un incremento en el número de casos de pacientes con metástasis óseas lo que unido a la incidencia de neoplasias óseas primarias, ha significado una aumento en la frecuencia absoluta de tumores óseas.

Es imprescindible un repaso y actualización de las características imagenológicas de las principales patologías neoplásicas que afectan al hueso y que son atendidas en el Hospital "Juan Tanca Marengo" del Instituto Oncológico Nacional Solca, una Institución especializada en el manejo terapéutico de pacientes con patología oncológica ubicado en la ciudad de Guayaquil - Ecuador. El objetivo del estudio fue determinar las características imagenológicas de lesiones óseas metastásicas y compararlas con las lesiones óseas primarias en un grupo de pacientes.

\section{Materiales y Métodos}

El presente estudio es observacional, descriptivo, y transversal que fue realizado en el Departamento de Imagenología del ION Solca-Guayaquil, entre el 1 ro de enero del 2006 al 30 de enero del 2008. Se incluyeron pacientes en los que se realizó estudios imagenológicos por neoplasia ósea confirmada, ya sea primaria o metastásica. Se excluyeron casos con expediente clínico incompleto que impedía la recolección de información.

Se indagó sobre variables como ubicación, margen, reacción perióstica, opacidad, mineralización, tamaño, número de lesiones, afectación cortical y componente de tejido blando.

El procesamiento de la información se efectuó utilizando el paquete estadístico SPSS 18. Para el análisis estadístico se emplearon promedios y desviación estándar en el caso de las variables numéricas y el cálculo de frecuencias relativas y absolutas en el caso de las variables categóricas.

\section{Resultados}

Se incluyeron en el estudio 100 casos, con edades de 0 a 69 años. La mayoría de los pacientes con tumor primario fueron incluidos en el grupo de 10 a 19 años y los tumores metastásicos en el de 40 a 49 años. El promedio de edad para los primeros fue de $25.4 \pm$ 16 años y el de los segundos de $32.8 \pm 14$ años (Tabla 1). Entre los pacientes con metástasis óseas, la población de mujeres fue superior, mientras que entre los pacientes con tumores primarios los hombres fueron ligeramente superiores en número. En ambos grupos de pacientes, la tecnología empleada con mayor frecuencia para el diagnóstico imagenológico fue la TC (Tabla 2); el hueso más afectado fue el fémur (Tabla 3); el segmento más afectado fue la epífisis (Figura 1). 
Tabla 1. Pacientes con tumores y metástasis óseas clasificados por edad

\begin{tabular}{|l|c|c|}
\hline Edad (años) & $\begin{array}{c}\text { Primario } \\
\mathrm{n}=84\end{array}$ & $\begin{array}{c}\text { Metastásico } \\
\mathrm{n}=16\end{array}$ \\
\hline $\mathbf{0 - 9}$ & $8(9.5 \%)$ & $1(6.2 \%)$ \\
\hline $\mathbf{1 0 - 1 9}$ & $35(41.7 \%)$ & $3(18.8 \%)$ \\
\hline $\mathbf{2 0 - 2 9}$ & $17(20.2 \%)$ & $3(18.8 \%)$ \\
\hline $\mathbf{3 0 - 3 9}$ & $8(9.5 \%)$ & $2(12.5 \%)$ \\
\hline $\mathbf{4 0 - 4 9}$ & $7(8.3 \%)$ & $5(31.2 \%)$ \\
\hline $\mathbf{5 0 - 5 9}$ & $5(6.0 \%)$ & $2(12.5 \%)$ \\
\hline $60-69$ & $4(4.8 \%)$ & $0(0 \%)$ \\
\hline
\end{tabular}

Figura 1. Pacientes con Tumores y Metástasis óseas clasificados por ubicación ósea.

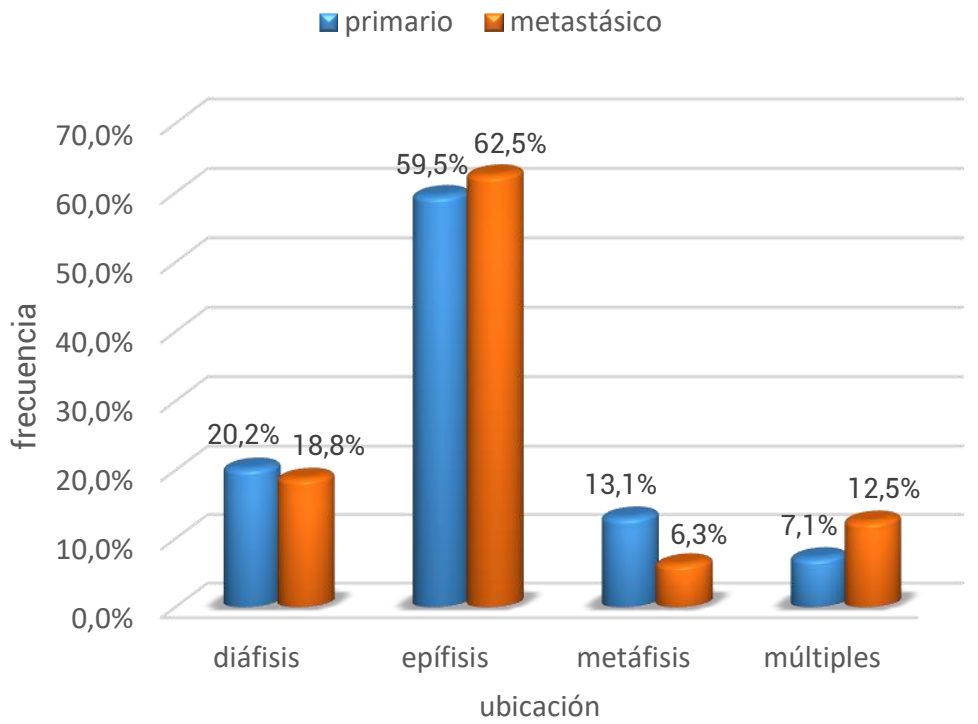

Tabla 2. Pacientes con Tumores y Metástasis óseas clasificados por tecnología de imágenes empleadas en el diagnóstico

\begin{tabular}{|l|c|c|}
\hline Estudio & $\begin{array}{c}\text { Primario } \\
\mathrm{n}=\mathbf{8 4}\end{array}$ & $\begin{array}{c}\text { Metastásico } \\
\mathrm{n}=16\end{array}$ \\
\hline RM & $5(6.0 \%)$ & $2(12.5 \%)$ \\
\hline RM+RX & $1(1.2 \%)$ & $0(0 \%)$ \\
\hline RM+TC & $2(2.4 \%)$ & $0(0 \%)$ \\
\hline RX & $22(26.2 \%)$ & $2(12.5 \%)$ \\
\hline RX+TC & $1(1.2 \%)$ & $0(0 \%)$ \\
\hline TC & $53(63.1 \%)$ & $12(75 \%)$ \\
\hline
\end{tabular}

RM: Resonancia Magnética, Rx: radiografía, TC: Tomografía. 
Tabla 3. Pacientes con Tumores y Metástasis óseas clasificados por hueso afectado

\begin{tabular}{|l|c|c|}
\hline Hueso afectado & $\begin{array}{c}\text { Primario } \\
\mathbf{n = 8 4}\end{array}$ & $\begin{array}{c}\text { Metastásico } \\
\mathrm{n}=16\end{array}$ \\
\hline Columna vertebral & $2(2.4 \%)$ & $2(12.5 \%)$ \\
\hline Cráneo & $5(6.0 \%)$ & $0(0 \%)$ \\
\hline Cúbito & $1(1.2 \%)$ & $0(0 \%)$ \\
\hline Fémur & $36(42.0 \%)$ & 8 \\
\hline Huesos de la mano & $2(2.4 \%)$ & $0(0 \%)$ \\
\hline Húmero & $10(11.9 \% 9$ & 2 \\
\hline Omóplato & $3(3.6 \%)$ & $0(0 \%)$ \\
\hline Pelvis & $10(11.9 \%)$ & 3 \\
\hline Peroné & $1(1.2 \%)$ & $0(0 \%)$ \\
\hline Radio & $1(1.2 \%)$ & $0(0 \%)$ \\
\hline Tibia & $13(15.5 \%)$ & $1(6.3 \%)$ \\
\hline
\end{tabular}

Tabla 4. Características radiológicas de los tumores y metástasis óseas

\begin{tabular}{|c|c|c|c|}
\hline \multicolumn{2}{|l|}{ Variable } & $\begin{array}{c}\text { Primario } \\
(n=84)\end{array}$ & $\begin{array}{l}\text { Metastásico } \\
\quad(n=16)\end{array}$ \\
\hline \multirow[t]{3}{*}{ Margen } & Poco definido & $59(70.2 \%)$ & $14(87.5 \%)$ \\
\hline & Medio definido & $9(10.7 \%)$ & $2(12.5 \%)$ \\
\hline & Bien definido & $16(19.0 \%)$ & $0(0 \%)$ \\
\hline \multirow[t]{6}{*}{$\begin{array}{l}\text { Reacción } \\
\text { perióstica }\end{array}$} & $\begin{array}{l}\text { Espiculado } \\
\text { multilaminal }\end{array}$ & $4(4.8 \%)$ & $2(12.5 \%)$ \\
\hline & Interrupción regional & $11(13.1 \%)$ & $2(12.5 \%)$ \\
\hline & Múltiple & $29(34.5 \%)$ & $10(62.4 \%)$ \\
\hline & Ninguno & $27(32.1 \%)$ & $1(6.3 \%)$ \\
\hline & Sólida & $1(1.2 \%)$ & $0(0 \%)$ \\
\hline & Triángulo de Codman & $12(14.3 \%)$ & $1(6.3 \%)$ \\
\hline \multirow[t]{2}{*}{ Opacidad } & Opaco & $11(13.1 \%)$ & $1(6.3 \%)$ \\
\hline & Radiolúcido & $73(86.9 \%)$ & $15(93.7 \%)$ \\
\hline \multirow[t]{2}{*}{ Patrón } & trabeculado & $82(97.6 \%)$ & $15(93,8 \%)$ \\
\hline & radial & $2(2.4 \%)$ & $1(6.3 \%)$ \\
\hline \multirow[t]{3}{*}{ Tamaño } & $1-5$ & $64(76.2 \%)$ & $15(93.7 \%)$ \\
\hline & $6-10$ & $16(19.0 \%)$ & $1(6.3 \%)$ \\
\hline & $>10$ & $4(4.8 \%)$ & $0(0 \%)$ \\
\hline \multirow[t]{3}{*}{ Número } & $1-5$ & $80(95.2 \%)$ & $16(100 \%)$ \\
\hline & $6-10$ & $2(2.4 \%)$ & $0(0 \%)$ \\
\hline & $>10$ & $2(2.4 \%)$ & $0(0 \%)$ \\
\hline Componente & sí & $33(39.3 \%)$ & $3(20.0 \%)$ \\
\hline
\end{tabular}

Las metástasis óseas y los tumores primarios por lo general presentaban márgenes de la lesión poco definidos; y cuando se presentó reacción perióstica, esta fue múltiple. Pero en 
el grupo que generalmente no se presentó reacción perióstica con mayor frecuencia fue entre los pacientes con tumores primarios. Al estudio imagenológico, el grado de opacidad de la lesión fue radiolúcido con mayor frecuencia para el grupo de tumores óseos primarios como para el grupo de metástasis óseas.

Ambos grupos tuvieron un patrón de mineralización trabeculado en la mayoría de los casos; la mayoría tenía una lesión que medía entre 1 a $5 \mathrm{~cm}$. El promedio de tamaño para el primer grupo fue de $5.6 \pm 5.6 \mathrm{~cm}$ y para los segundos fue de $4.7 \pm 1.5 \mathrm{~cm}$. Para ambos estudios, la mayoría presentó entre 1 y 5 lesiones. La media del número de lesiones para los primeros fue de $1.6 \pm 1$ y para los segundos de $1.1 \pm 0.3$. Entre los pacientes con tumores óseos primarios, la mayoría no tuvo afectación cortical, mientras que, entre los pacientes con metástasis óseas, se presentó con mayor frecuencia compromiso de la corteza. Para ambos grupos, la presencia de componente de tejido blando en la lesión fue poco frecuente (Tabla 4).

\section{Discusión}

El presente estudio registra que la mayoría de tumores fueron diagnosticados entre los 10 a 19 años de edad, la mayoría de casos (84 \%) fueron tumores primarios. Los sarcomas representan $1 \%$ de todos los cánceres en adultos, a nivel mundial el $10 \%$ de los cánceres en los niños, y $8 \%$ de cáncer en los adolescentes y adultos jóvenes. La incidencia de osteosarcoma es ligeramente mayor en hombres que en mujeres. En los hombres, es de 5.2 por millón por año. En las mujeres, la incidencia es de 4.5 por millón por año [9].

En los Estados Unidos, la incidencia de osteosarcoma es de 400 casos por año (4.8 por millón de habitantes en menores de 20 años). El diagnóstico apropiado de los tumores óseos requiere un examen cuidadoso de todas las fuentes de información disponibles, incluyendo la historia del paciente, el examen físico, las radiografías simples y otras técnicas de imagen, como la TC y la RM. Cada opción de imagen tiene sus propios puntos fuertes, que deben ser considerados durante el análisis.

El ejercicio diagnóstico de diferenciar una lesión primaria ósea de una lesión metastásica depende de los antecedentes clínicos del paciente. En este estudio se demuestra que las características imagenológicas de los tumores óseos y las metástasis óseas no son específicas para el tipo de lesión. Aunque la herramienta de diagnóstico más exacto disponible para el clínico es la biopsia de hueso, no demuestra pruebas de la progresión o la agresividad de la lesión.

Los tumores óseos primarios presentan generalmente un conjunto específico de síntomas y apariencias radiográficas, pero otras enfermedades óseas no neoplásicas también pueden presentar síntomas similares. Por lo tanto, el diagnóstico diferencial no siempre es fácil y requiere la máxima atención.

Los tumores benignos son relativamente comunes, y pocos tienen la capacidad y la tendencia a la degeneración maligna. La mayoría de los tumores benignos permanecen asintomáticos hasta que su presencia se indica por una lesión trivial o accidental. Los 
tumores óseos primarios malignos más comúnmente diagnosticado incluyen: mieloma múltiple, osteosarcoma, condrosarcomas, y el sarcoma de Ewing [13].

Los tumores óseos, como osteosarcomas y sarcoma de Ewing, son tumores malignos típicos de los adolescentes y adultos jóvenes [14], mientras que el mieloma y el condrosarcoma son frecuentes en la adultez.

Con respecto a las metástasis de carcinomas, éstas son los tumores malignos más comunes que afectan el hueso. El cáncer de próstata, mama y pulmón son las neoplasias más comunes en los adultos y son los tumores que más comúnmente hacen metástasis a los huesos. Por otra parte, las metástasis óseas afectan a más del $60 \%$ de pacientes con cáncer de mama y de próstata en estadios avanzados. El carcinoma del riñón, tiroides y el melanoma son tumores que de forma común metastatizan al hueso [15].

Las metástasis óseas son usualmente multifocales con predilección por sitios hematopoyéticos medulares, en los huesos largos proximales y el esqueleto axial (vértebras, pelvis, costillas y cráneo). La continua rotación dinámica de la matriz del hueso y la médula ósea proporciona un terreno fértil para que las células tumorales utilicen los vastos recursos disponibles (células, factores de crecimiento, citocinas y receptores), proliferen y posteriormente metastaticen [15]. Las células cancerosas provocan un microambiente óseo en el que se rompió el equilibrio fisiológico entre la resorción ósea y la formación de desarrollo de lesiones líticas, blásticas o mixtas [16].

Alrededor del $25 \%$ de los pacientes son asintomáticos, el diagnóstico se realiza mediante exámenes prescritos por otras razones o durante la estadificación del tumor primario. El $75 \%$ de las metástasis en los huesos restantes son responsables de diferentes complicaciones clínicas como dolor, fracturas patológicas, compresión de la médula espinal, depresión de la médula ósea y el deterioro del estado funcional. El dolor es el síntoma más frecuente, el cual disminuye la movilidad del paciente, aumenta la ansiedad y la depresión, predispone a otras complicaciones como infecciones pulmonares, la trombosis venosa, las úlceras cutáneas, lo que reduce la calidad de vida. La frecuencia de otras complicaciones depende de la naturaleza de las lesiones óseas, su sitio, y su tratamiento [17].

Las lesiones líticas (más frecuentes en el cáncer de mama, tiroides, riñón y pulmón) predisponen a complicaciones graves, como fracturas patológicas e hipercalcemia. La prevalencia de las lesiones osteoblásticas, como cáncer de próstata, predispone con una frecuencia menor a estas complicaciones. La hipercalcemia es inusual en estos pacientes, en cambio, es más frecuente observar una hipocalcemia [10]. Debido a la gran variedad de signología radiológica se recomienda realizar un estudio para medir la variación de la interpretación interobservador e intraobservador al momento de valorar imágenes de diagnóstico para evaluar lesiones tumorales óseas en esta Institución.

En el caso de la afectación cortical parece existir una tendencia a que esta característica se encuentre con mayor frecuencia en pacientes con metástasis óseas, pero no se configura una diferencia significativa. 


\section{Conclusiones}

Las características imagenológicas de los tumores óseos y las metástasis óseas no son específicas para el tipo de lesión. En el caso de la afectación cortical parece existir una tendencia a que esta característica se encuentre con mayor frecuencia en pacientes con metástasis óseas, pero no se configura una diferencia significativa.

\section{Agradecimientos}

Quiero agradecer al personal del Instituto Oncológico Solca-Guayaquil por su colaboración en el presente estudio.

\section{Información adicional}

\section{Abreviaturas}

RM: Resonancia Magnética.

Rx: Radiografía.

TC: Tomografía.

Solca: Sociedad de Lucha Contra el Cáncer.

Nota del Editor

La Revista Oncología Ecu permanece neutral con respecto a los reclamos jurisdiccionales en mapas publicados y afiliaciones institucionales.

\section{Archivos Adicionales}

Ninguno declarado por la autora.

\section{Fondos}

Los fondos para la presente investigación fueron propios de la autora del presente artículo.

\section{Disponibilidad de datos y materiales}

Existe la disponibilidad de datos bajo solicitud a la autora de correspondencia. No se reportan otros materiales.

\section{Contribuciones de los autores}


EMP idea de investigación, revisión bibliográfica, compilación de datos, escritura académica.

Aprobación de ética y consentimiento para participar

No aplica ya que es un estudio de revisión de historias clínicas.

\section{Consentimiento para publicación}

No aplica.

Información de la autora

Elena Mariño Parra, Médica Imagenóloga del Hospital Roberto Gilbert. https://orcid.org/0000-0002-0936-2732

Revisiones por pares

Acceda a la revisión de pares académicos en el siguiente enlace: https://publons.com/review/2898998

Abreviaturas en la referencias

DOI: Digital Object

Identifier

PMID: PubMed Identifier

SU: Short URL

\section{Referencias}

1. Kricun ME. Radiographic evaluation of solitary bone lesions. Orthop Clin North Am 1983;14:39-64. PMID: www.ncbi.nlm.nih.gov/pubmed/6338449

2. Madewell JE, Ragsdale BD, Sweet DE. Radiologic and pathologic analysis of solitary bone lesions. I. Internal margins. Radiol Clin North Am 1981; 19: 715-748. PMID: www.ncbi.nlm.nih.gov/pubmed/7323290

3. Priolo F, Cerase A. The current role of radiography in the assessment of skeletal tumors and tumor-like lesions. Eur J Radiol 1998;27(suppl 1):S77-S85. PMID: www.ncbi.nlm.nih.gov/pubmed/9652506

4. Ragsdale BD, Madewell JE, Sweet DE. Radiologic and pathologic analysis of solitary bone lesions. II. Periosteal reactions. Radiol Clin North Am 1981;19:749-783. PMID: www.ncbi.nlm.nih.gov/pubmed/7323291

5. Sweet DE, Madewell JE, Ragsdale BD. Radiologic and pathologic analysis of solitary bone lesions. III. Matrix patterns. Radiol Clin North Am 1981;19:785-814. PMID:

www.ncbi.nlm.nih.gov/pubmed/7323292

6. Brown KT, Kattapuram SV, Rosenthal DI. Computed tomography analysis of bone tumors: patters of cortical destruction and soft tissue extension. Skeletal Radiol 1986;15:448-451. PMID: www.ncbi.nlm.nih.gov/pubmed/3464100 
7. Hayes CW, Conway WF, Sundaram M. Misleading aggressive MR imaging appearance of some benign musculoskeletal lesions. RadioGraphics 1992;12:1119-1136. URL: http://pubs.rsna.org/doi/pdf/10.1148/radiographics.12.6.1439015

8. Ma LD, Frassica FJ, Scott WW, Fishman EK, Zerhouni EA. Differentiation of benign and malignant musculoskeletal tumors: potential pitfalls with MR imaging. RadioGraphics 1995;15:349-366. URL: http://pubs.rsna.org/doi/pdf/10.1148/radiographics.15.2.7761640

9. Seeger LL, Dungan DH, Eckardt JJ, Bassett LW, Gold RH. Nonspecific findings on MR imaging: the importance of correlative studies and clinical information. Clin Orthop Relat Res 1991;270:306-312. PMID: www.ncbi.nlm.nih.gov/pubmed/1884555

10. Coleman RE. Skeletal complications of malignancy. Cancer. 1997; 80: 1588-1594. PMID: https://www.ncbi.nlm.nih.gov/pubmed/9362426

11. MSP Ecuador. Indicadores básicos de Salud. Ecuador 2006. Talleres Gráficos INEC. Quito, 2007

12. MSP Ecuador. Indicadores básicos de Salud Ecuador 2010. Talleres Gráficos INEC. Quito 2010.

13. Muir MJ, Bhandari M, Dunlop RB. Common primary tumors of bone. Can J Diagn. 2001;(2):121-13. URL: http://www.stacommunications.com/journals/diagnosis/2001/05_May/commonbone.pdf

14. Orbach D, Glorion C, Mary $\mathrm{P}$, et al. Malignant tumors of the locomotor apparatus in children: an intermediate prognosis. Rev Prat. 2007;57:1080-1086. PMID: www.ncbi.nlm.nih.gov/pubmed/17844801

15. Virk SM, Lieberman JR. Tumor metastasis to bone. Arthritis Research \& Therapy. 2007;9(Suppl1):1-7. PMID: www.ncbi.nlm.nih.gov/pmc/articles/PMC1924520/pdf/ar2169.pdf

16. Ibrahim T, Flamini E, Mercatali L. Pathogenesis of osteoblastic bone metastases from prostate cancer. Cancer. 2010 Mar 15;116(6):1406-18. URL:

http://onlinelibrary.wiley.com/doi/10.1002/cncr.24896/full

17. Ibrahim T, Farolfi A, Mercatali L. Metastatic bone disease in the era of bone targeted therapy: clinical impact. Tumori. 2013; 9(1): 1-124. PMID: www.ncbi.nlm.nih.gov/pubmed/23548992 